\title{
Hablemos de estudiantes digitales $y$ no de nativos digitales
}

\author{
Eliana E. Gallardo Echenique ${ }^{a}$ \\ Rebut: 20/03/2012 Acceptat: 08/05/2012
}

\section{Resumen}

En el presente artículo, se analiza el contexto de la discusión sobre los "nativos digitales" y su competencia digital. De la revisión literaria, dos conclusiones se pueden extraer. En primer lugar, no hay una definición absoluta de los nativos digitales que puede variar entre los individuos, sociedades, regiones y naciones, y también a través del tiempo. En segundo lugar, hay un número de otras variables que la edad para predecir la naturaleza del uso de las tecnologías digitales en los estudiantes. En base a los resultados, la revisión literaria sobre los nativos digitales demuestra que a pesar de estar familiarizados con el uso de las tecnologías digitales y que sus habilidades digitales son altas, la competencia digital - la capacidad de evaluar y aprender desde los recursos - puede ser menor que la de sus profesores.

Palabras claves: alfabetización digital, competencia digital, nativos digitales, era digital

\section{Let's talk about digital learners and not about digital natives}

\section{Abstract}

This article analyzed the context of discussion about "digital natives" and their digital competence. Two findings can be drawn from this review. First, there is no absolute definition of digital native: it will vary amongst individuals, societies, regions and nations, and also over time. Second, there are a number of variables other than age that may predict the nature of students' use digital technologies. On the basis of the findings, the analysis of the digital native literature demonstrates that despite their digital confidence and digital skills are high, their digital competence - the ability to assess and learn from resources - may be much lower than those of their digitally teachers.

Keywords: digital literacy, digital competence, digital natives, digital era

\footnotetext{
${ }^{a}$ Departament de Pedagogia. Universitat Rovira i Virgili - Tarragona
} 


\section{Introducción}

Las tecnologías de la información y comunicación (TIC) han desempeñado y continúan desempeñando un papel fundamental en la configuración de nuestra sociedad y cultura. Su utilización ha provocado modificaciones en todos los campos del saber a ritmo vertiginoso, proporcionando una serie de herramientas y contextos de comunicación y de aprendizaje, de enorme potencialidad y ha obligado a redefinir incluso el concepto de la realidad, a partir de la posibilidad de construir nuevas realidades. Investigadores como Cabero y Aguaded (2003: 12) afirman que las tecnologías de la información y comunicación, en una sociedad marcada consecuentemente por ellas, están modificando nuestros estilos de vida, nuestras pautas de conducta, nuestros hábitos de ocio y de trabajo.

La tecnología digital se hace presente en todas las áreas de actividad y colabora con los cambios que se producen en el trabajo, la familia y la educación, entre otros (UNESCO, 2011: 9). Su introducción en el ámbito educativo viene enmarcada por una situación de cambios en los docentes y estudiantes, en los entornos o escenarios de aprendizaje, en sus modelos y concepciones. En la actualidad, las TIC permiten que los procesos de enseñanza sean adaptados según las características personales de los estudiantes, a sus necesidades de estilos de aprendizaje y en cierta medida supone que pasemos de una cultura de la enseñanza, a una cultura del aprendizaje, ya que la mejor forma de aprender, no es reproduciendo los conocimientos, sino construyéndolos a través de una actitud activa por parte del estudiante, y no pasiva en la interacción con la información (Cabero, 2006: 8).

En los últimos años, se ha producido una revolución en torno a las tecnologías de la información y comunicación que han originado el planteamiento de la era digital, es decir, de una época en la que la informática y la telemática están produciendo transformaciones en la sociedad, educación, cultura, ciencia, industria, comercio, entre otras actividades. En efecto, es la digitalización la que provoca el desarrollo exponencial de las TIC. Estamos en los albores de un nuevo mundo - el digital - que aparece desde que las TIC han revolucionado la forma de relacionarnos y de hacer las cosas.

La era digital plantea retos y transformaciones en el ámbito educativo (instituciones, profesores, estudiantes, familia), en las estrategias pedagógicas y didácticas que por lo general se llevan a cabo en el aula, lugar social que no es ajeno a los influjos de la tecnología. Esta era requiere que las personas estén en continua, constante y libre exposición a ambientes digitales sin restricciones; y para poder utilizar y apropiarse de los equipos en todo lugar y momento, es preciso construir y vivir en un mundo digital (Irigoyen, 2000a, 2000b; Camacho \& González, 2008).

La era digital pone en evidencia la necesidad de una nueva definición de roles, especialmente, para los estudiantes y los docentes, a la vez que implica replantear el proceso educativo en torno a la forma en que los estudiantes procesan la información y no sólo en torno a las herramientas o recursos TIC. En las últimos años, los estudiantes han cambiado radicalmente - no son las mismas personas para las 
que nuestro sistema educativo fue diseñado para enseñar - debido a la rápida diseminación de la tecnología digital. Las TIC están propiciando en los estudiantes una visión de mundo distinta, generando nuevas habilidades y/o competencias, e impactando su vida social y académica. Los cambios en la percepción de espacio y tiempo, las nuevas estrategias cognitivas y la interacción permanente con dispositivos tecnológicos son características propias de los estudiantes.

Por otro lado existe, como nunca antes, una brecha generacional entre los principales agentes de la comunidad educativa, generada por concepciones, paradigmas y visiones propias de cada generación. Sin embargo, en un mundo cambiante, impactado por los rápidos avances de la ciencia y la tecnología, las brechas entre docentes y estudiantes no sólo se limitan a las propias de las generaciones, sino que incluyen diferencias en el uso, manejo y acceso de la tecnología. Esta generación se involucran con el mundo de manera diferente: son neurológicamente diferentes, piensan y procesan información de manera diferente utilizando diversas partes del cerebro que las personas de nuestra generación y como resultado tienen diferentes estilos y preferencias de aprendizaje (Jukes, 2009; Jukes, Mccain, \& Crockett, 2010).

Por este motivo, el objetivo de este artículo es contribuir con la discusión sobre si estos nuevos estudiantes digitales (también conocidos como nativos digitales) y sus habilidades de la competencia digital que se consideran necesarias para el aprendizaje eficaz y consciente en los emergentes entornos digitales emergentes. Para alcanzar este objetivo, es necesario utilizar el método de la revisión literaria para fusionar, resumir y revisar la literatura. El énfasis se pone en la literatura que emergió después del 2000 en adelante.

\section{Estudiantes digitales y no nativos digitales}

En muchos países desarrollados, la mayoría de los adolescentes hacen uso intensivo de la tecnología digital, especialmente de Internet (Ben-David Kolikant, 2010: 1384). La mayoría de estos estudiantes, quienes nacieron aproximadamente entre 1980 y 1994 (Oblinger \& Oblinger, 2005), representan las primeras generaciones que crecieron con esta nueva tecnología y se han caracterizado por su familiaridad y confianza con respecto a las TIC. Los estudiantes de esta nueva generación "han crecido en un mundo digital y esperan utilizar estas herramientas para sus entornos avanzados de aprendizaje" (Bajt, 2011: 54). Ellos han pasado toda su vida rodeados y usando computadoras, videojuegos, reproductores de música digital, cámaras de video, teléfonos celulares, i-pods, Internet, mensajería instantánea, mensajes de texto, multimedia y otras herramientas de la era digital que son parte integral de sus vidas. Hoy piensan y procesan la información de manera diferente a como lo hacían sus predecesores, porque sus patrones de pensamiento han cambiado. Pero, ¿cómo debemos llamar estos "nuevos" estudiantes de hoy? Esta generación ha recibido varios nombres que enfatizan su afinidad y tendencia al momento de utilizar tecnología digital como: 


\begin{tabular}{|l|l|l|}
\hline \multicolumn{1}{|c|}{ Denominación } & \multicolumn{1}{c|}{ Autor } & Año \\
\hline Millenials & Howe y Strauss & 1991 \\
& Lancaster y Stillman & 2002 \\
& Martin y Tulgan & 2002 \\
& Oblinger y Oblinger & 2005 \\
\hline Generación Digital & Tapscott & 1998 \\
\hline Generación Net & Tapscott & 1998 \\
& Oblinger y Oblinger & 2005 \\
\hline Nexters & Zemke, Raines y Filipczak & 1999 \\
\hline Nativos e inmigrantes digitales & Prensky & 2001 \\
\hline Generación Instant Message o SMS & Lenhart, Rainie y Lewis & 2001 \\
\hline Generación Y & Lancaster y Stillman & 2002 \\
& Jorgensen & 2003 \\
& Oblinger y Oblinger & 2005 \\
& Weiler & 2005 \\
\hline Homo Zappiens & McCrindle & 2006 \\
\hline Gamer Generation & Veen & 2003 \\
\hline New Millennial Learners & Carstens y Beck & 2005 \\
\hline Generación C & Pedró & 2006 \\
\hline Google Generation & Duncan-Howell y Lee & 2007 \\
\hline Digital Learners & Rowlands y Nicholas & 2008 \\
\hline Generación F / Facebook Generation & Bullen & 2008 \\
\hline i-Generation & Kitsis & 2008 \\
\hline Visitantes y Residentes & Hamel & 2009 \\
\hline & Rosen et al. & 2010 \\
\hline & White & 2010 \\
\hline
\end{tabular}

Tabla 1: Denominaciones del nuevo perfil del estudiante. De Digital learners: la competencia digital de los estudiantes universitarios., por Gisbert, \& Esteve, 2011, La Cuestión Universitaria, 7, p. 52.

De acuerdo con la literatura, tres de los términos más comunes son: Generación Net (Net Generation), Millennials y nativos digitales (Jones \& Czerniewicz, 2010: 317). Cada manera de describir a esta nueva generación lleva consigo algunas características distintas, pero en general, los términos se utilizan indistintamente (Jones et al., 2010: 723).

El término "nativos digitales" fue acuñado por Marc Prensky en el ensayo "Digital Natives, Digital Immigrants", publicado en 2001. Prensky (2001a, 2001b) utiliza los términos nativo digital e inmigrante digital para distinguir entre aquellos quienes han crecido familiarizados con múltiples tecnologías y aquellos quienes nacieron antes de que el nuevo mundo digital comenzara. Según el autor, los estudiantes de hoy son todos "nativos" del lenguaje digital de los ordenadores, videojuegos e Internet (Prensky, 2001a: 1). El principal argumento de Prensky es que este nuevo grupo de estudiantes quienes han accedido a las universidades son esencialmente diferentes que sus profesores, debido al uso constante y frecuente de las tecnologías. Ellos usan estas herramientas como extensiones de sus cuerpos y mentes, con fluidez las incorporan en sus rutinas diarias (Prensky, 2005), han aprendido el lenguaje de la tecnología, y se comunican instantáneamente con sus compañeros. Estos estudiantes, 
al igual que todos los "nativos", se adaptan rápidamente a los cambios en su entorno y buscan nuevas formas de incorporar la última tecnología en sus aceleradas vidas. Según Palfrey, Gasser, Simun, \& Barnes (2009: 79) nativos digitales son "los jóvenes que crecen en el mundo digital con el acceso a las tecnologías y las habilidades para utilizarlas en formas sofisticadas".

Para Jones \& Czerniewicz (2010: 317) el término Generación Net se compone de los nativos digitales. Generación Y, también conocido como generación del Milenio - en inglés Millennial generation (los estudiantes nacidos después de 1980), es la generación más grande desde la generación del baby boom (Norum, 2008; Coomes, \& DeBard, 2004). La generación $Y$ ha crecido con la tecnología y "es una de las primeras generaciones que tienen la tecnología y el Internet desde una edad muy temprana - ellos son significativamente más propensos que los usuarios mayores de Internet para crear blogs, descargar música, enviar mensajes instantáneos, y jugar juegos en línea" (Djamasbi, Siegel, \& Tullis, 2010: 309). Estudiantes de la Generación Net han crecido en un mundo digital y esperan utilizar estas herramientas a su favor, en entornos de aprendizaje (Bajt, 2011: 54).

En una reciente investigación "Pew research study" (Taylor, y Keeter, 2010: 1) - que explora los comportamientos, valores y opiniones de los adolescentes de la nueva generación - "generaciones, como las personas, tienen personalidades, y Millennials son confiados, auto-expresivos, liberales, optimistas y abiertos al cambio". Según el estudio de investigación realizado por la Fundación de la Familia Henry J. Kaiser (Henry J. Kaiser Family Foundation, 2010), en promedio, Millennials pasan 7,5 horas diarias saturados de información proveniente de medios impresos, electrónicos, digitales y de comunicación. Ellos escuchan y graban música, ven, crean y publican contenidos en Internet, juegan videojuegos, ven televisión, hablan por teléfonos móviles y envían mensajes instantáneos cada día.

La noción de nativo digital es un término que "rápidamente ganó reconocimiento y aprobación generalizada" (Kennedy, et al., 2010: 333). Sin embargo, las afirmaciones más importantes en el discurso sobre los nativos digitales no surgen de la literatura académica, debido que algunos aparecen en la prensa popular y no especializada; son investigaciones financiadas por la empresa privada o carecen del rigor académico suficiente para ser una investigación empírica. Para Bullen \& Morgan (2011), quienes vienen realizando desde hace unos años, una investigación de carácter internacional, en seis países y en diversas instituciones, sugieren el término estudiantes digitales (digital learners) porque los estudiantes de hoy no se ajustan al estereotipo representado en el discurso de los nativos digitales. Para los autores es una cuestión social y no generacional, y sus implicaciones para la educación aún necesitan mayor estudio a profundidad.

¿Cómo son los estudiantes digitales? Los nuevos estudiantes digitales están inmersos en tecnología y ellos esperan emplear las herramientas digitales como parte de su experiencia educativa (D'Amico, 2010/2011). Según Jukes, Mccain, \& Crockett (2010: 10) están conectados por multimedia. Debido a los efectos del bombardeo digital, 
ellos piensan de forma gráfica y, por lo tanto, ya son estudiantes "visuales o kinestésicos".

Para el International Learning Advisory Board (2008), estos jóvenes comparten las siguientes características:

- Les gusta tener el control. No quieren estar sujeto a los horarios tradicionales, y no necesariamente quieren estar en un aula para aprender o en una oficina para trabajar. En cambio, prefieren utilizar la tecnología para estudiar en cualquier momento del día o de la noche y, a su manera, trabajar desde cualquier lugar del mundo.

- Les gusta escoger. Utilizan la tecnología para realizar tareas de maneras nuevas y creativas. Su necesidad por métodos alternativos para realizarlas presenta retos cuando se usan mediciones tradicionales para definir la productividad.

- Son orientados al grupo y a lo social. Expuestos al mundo a través de los medios de comunicación, ellos constantemente están conectados a través de las redes sociales. En línea, buscan oportunidades de identificarse con otras personas, uniéndose a las comunidades y las asociaciones con sus pares en todo el mundo. Son altamente colaborativos; comparten lo que han aprendido con otras personas lo que contribuye a crear sus propias identidades personales.

- Son inclusivos. Fueron enseñados a ser tolerantes con todas las razas, religiones y orientaciones sexuales. No están limitados por las informaciones disponibles en su biblioteca local o por búsquedas lineales sobre específicos temas en las enciclopedias. En cambio, utilizan el Internet para buscar información a nivel mundial y utilizan los hipervínculos para desviar la atención de sus búsquedas originales y aprender sobre nuevos temas.

- Son usuarios expertos de la tecnología digital. Esta generación es la primera en estar rodeada por los medios digitales de comunicación. Las TIC siempre han sido parte de sus vidas y debido al acceso, ellos se sienten atraídos a ellas. De hecho, pueden realizar muchas funciones con los teléfonos móviles, dispositivos portátiles y otros equipos inalámbricos que los que pueden realizar con los ordenadores tradicionales. Además de utilizar las funciones de calendario de estos dispositivos para planificar sus vidas, a menudo prefieren la comunicación mediada por el ordenador y han desarrollado su propio lenguaje, que consta de acrónimos, en especial en el mundo anglosajón, como "LOL" ("Laughing out loud"), "ATM" ("At the moment"), "BTW" ("By the way") y otras jergas de Internet. La tecnología actual les permite expresar sus opiniones de manera que no estaban disponibles en el pasado. 
- Piensan de manera diferente. La tecnología en sí no les sorprende como a las generaciones anteriores que primero tratan de comprender cómo funciona la nueva tecnología; esta generación la acepta, la adapta y la utiliza.

- Son más propensos a tomar riesgos. El boom del punto.com a finales de los 90 se produjo gracias a esta generación que compartían una mentalidad común: "Si esto no funciona, vamos a intentarlo de nuevo".

- Ellos valoran el tiempo libre. Desean su propio tiempo para estar libres y que este tiempo sea dictado de acuerdo a sus propios términos.

Para la Asociación de Bibliotecarios de Nueva York (New York Library Association, 2008), los estudiantes digitales utilizan sus habilidades, recursos y herramientas para:

1. Investigar, pensar críticamente y adquirir conocimientos.

2. Sacar conclusiones, tomar decisiones con fundamento, aplicar conocimientos a situaciones nuevas y crear nuevos conocimientos.

3. Compartir conocimientos y participar ética y productivamente.

4. Desarrollar la comprensión por las ideas e información para alcanzar el crecimiento personal.

¿Qué es lo que desean estos estudiantes de la escuela? Sobre la base de las entrevistas realizadas a más de mil estudiantes de diferentes edades y estratos económicos, sociales, intelectuales, con el objetivo de conocer sus preferencias, Prensky (2010) concluye:

- No desean recibir "sermones".

- Desean ser respetados, que confíen en ellos, y que sus opiniones sean consideradas y valiosas.

- Desean seguir sus propios intereses y pasiones.

- Desean crear, utilizando las herramientas de su tiempo.

- Desean realizar proyectos y trabajos en grupo y de manera colaborativa.

- Desean tomar decisiones y compartir el control.

- Desean conectarse con sus compañeros para expresar y compartir sus opiniones durante la clase y alrededor del mundo.

- Desean cooperar y competir entre sí.

- Desean una educación que no sólo sea relevante sino real. 
En un reciente estudio llevado a cabo por el 21st Century Fluency Project, se mencionan 8 preferencias de aprendizaje de los estudiantes digitales (Tabla 2) que fueron comparadas con las de sus profesores.

\begin{tabular}{|c|c|}
\hline Estudiantes digitales prefieren: & Educadores prefieren: \\
\hline $\begin{array}{l}\text { recibir información de forma rápida a partir } \\
\text { de múltiples recursos multimedia }\end{array}$ & $\begin{array}{l}\text { recibir información de manera lenta y } \\
\text { controlada a partir de recursos limitados. }\end{array}$ \\
\hline procesamiento paralelo y multitarea & $\begin{array}{l}\text { procesamiento singular y tareas únicas o } \\
\text { limitadas }\end{array}$ \\
\hline $\begin{array}{l}\text { procesamiento de imágenes, colores, } \\
\text { sonidos y video antes que texto }\end{array}$ & $\begin{array}{l}\text { proporcionar texto antes que imágenes, } \\
\text { sonidos y video }\end{array}$ \\
\hline $\begin{array}{l}\text { acceso aleatorio a la hipervinculado a la } \\
\text { información multimedia }\end{array}$ & $\begin{array}{l}\text { proporcionar información de manera lineal, } \\
\text { lógica y secuencial }\end{array}$ \\
\hline $\begin{array}{l}\text { conectarse a la red de forma simultánea con } \\
\text { muchos otros }\end{array}$ & $\begin{array}{l}\text { estudiantes que trabajen de forma } \\
\text { independiente antes que se conecten a la red } \\
\text { e interactúen }\end{array}$ \\
\hline aprender "just-in-time" & enseñar "just-in-case" \\
\hline $\begin{array}{l}\text { gratificación instantánea y recompensas } \\
\text { inmediatas }\end{array}$ & gratificación diferida y recompensas tardías \\
\hline $\begin{array}{l}\text { aprendizaje que es relevante, activo, útil, al } \\
\text { instante y divertido }\end{array}$ & $\begin{array}{l}\text { sentirse obligados a enseñar según la guía } \\
\text { curricular y a realizar pruebas }\end{array}$ \\
\hline
\end{tabular}

Tabla 2: Preferencias de aprendizaje de los estudiantes digitales. De Understanding the Digital Generation: Teaching and Learning in the New Digital Landscape, por Jukes, McCain \& Crockett, 2010, p. 1114, (traducción y elaboración propia).

\section{Dos visiones confrontadas de un mismo concepto}

En la literatura, se asume que los estudiantes a veces se sienten facultados con respecto al aprendizaje debido a su familiaridad y al acceso a las TIC (Ben-David Kolikant, 2010: 1384).

Este tema ha generado controversia en la literatura. Por un lado, algunos investigadores académicos sostienen que los "nativos digitales" son usuarios avanzados de las nuevas tecnologías quienes analizan críticamente la información en línea (Frand, 2000; Arafeh et al., 2002; Gaston, 2006). De acuerdo con Virkus (2008: 266), estos nuevos estudiantes son: mejores recogiendo información, tomando decisiones rápidamente, multitareas, procesan en paralelo y piensan gráficamente en vez de textual; asumen la conectividad y ven el mundo a través de lentes de juegos y actividades lúdicas; tienen una diversidad de experiencias y necesidades, y están esperando respuestas y retroalimentación inmediatas; y están orientados a los logros y objetivos.

Por otro lado, la mayor parte de los investigadores académicos (Kennedy et al., 2008; Bennett, Maton \& Kervin, 2008; Brown \& Czerniewicz, 2010) demuestran que los "nativos digitales" parecen tener una superficial comprensión de las nuevas tecnologías, la usan para fines muy específicos y limitados, y poseen habilidades superficiales para buscar información y de análisis. En los últimos años, 
investigaciones empíricas sobre el uso y preferencias de los estudiantes de la Generación Net sobre las tecnologías en la educación superior reveló que "si bien la mayoría de los estudiantes regularmente usan tecnologías establecidas, tales como correo electrónico y buscadores, sólo un pequeño grupo de estudiantes utilizan las más avanzadas o recientes herramientas y tecnologías" (Kennedy et al., 2010: 333).

El proyecto de investigación de carácter internacional "Digital Learners in Higher Education" (http://digitallearners.ca/), que investiga cómo los estudiantes de educación superior en diferentes contextos institucionales y culturales piensan acerca de las TIC y cómo las utilizan en su vida social y educativa, ha encontrado que no hay una base empíricamente fidedigna para la mayoría de las afirmaciones que se han hecho acerca de la generación Net (Bullen, Morgan, \& Qayyum, 2011: 2). El estudio sugiere que no hay diferencias significativas entre los estudiantes de la Generación Net y quienes no lo son en términos de su uso de la tecnología, sus características de comportamiento y sus preferencias de aprendizaje. La investigación muestra que los nuevos usuarios, independientemente de la edad, comparten las siguientes características: poseen las habilidades que estas herramientas requieren, tienen acceso a ellas, saben cómo utilizarlas y conocen sus beneficios (Bullen \& Morgan, 2011: 60). Las afirmaciones que se realizan acerca de esta generación encajan en tres categorías: el uso generalizado e intensivo de las tecnologías digitales, el impacto de su uso sobre cómo esta generación accede y utiliza la información, la forma en que interactúan socialmente y cómo aprenden; y sus características especiales de comportamiento y estilos de aprendizaje de esta generación (Bullen, Morgan, \& Qayyum, 2011: 6).

Un estudio empírico más extenso (Kennedy et al. 2008) - realizado en 2006 con más de 2000 ingresantes al primer año de la universidad de Australia - ha comparado a los nativos e inmigrantes digitales con respecto al uso de la tecnología examinando qué herramientas utilizan y con qué frecuencia. Esta investigación demostró que no hay una diferencia fundamental entre los nativos e inmigrantes digitales y sugirió que las características de los nativos digitales sólo pueden encontrarse en una minoría de estudiantes. En un estudio más reciente llevado a cabo en dos universidades del Reino Unido, Margaryan et al. (2011: 439) "sugieren que las decisiones en torno al uso de las tecnologías para el aprendizaje no sólo deben estar basados en las preferencias de los estudiantes y sus prácticas actuales, sino en el entendimiento profundo sobre cuál es el valor educativo de estas tecnologías, y cómo pueden mejorar el proceso y los resultados de aprendizaje". Por lo tanto, es necesario alejarnos del discurso estereotípico generacional simplista y sin fundamento y desarrollar un conocimiento más minucioso al respecto. 


\section{Conclusiones}

A la luz de los resultados de las investigaciones, se ha demostrado la extensa diversidad teórica y literaria en torno al término "nativo digital" y cómo los autores e investigadores en un intento de acuñar una denominación, han proporcionado múltiples definiciones: algunas parecidas, otras bastantes distantes y en muchas de ellas redundantes. Además, sugerimos el término estudiante digital propuesto por Bullen y su equipo de investigación, porque recoge una visión más global del estudiante del siglo XXI.

Se tiene la creencia generalizada de que los nativos digitales muestran mejor disposición y capacidad para acercarse a las tecnologías. Sin embargo, la revisión literaria muestra lo contrario dado que hay un claro desequilibrio entre la seguridad con que las afirmaciones son formuladas y las pruebas de tales afirmaciones (Bennett et al., 2008). Al respecto, dos conclusiones se pueden extraer. En primer lugar, no hay una definición absoluta de los nativos digitales ya que puede variar entre los individuos, sociedades, regiones y naciones, y a través del tiempo. En segundo lugar, nacer en la era digital no implica una mayor competencia digital. Se ha encontrado que no hay suficiente evidencia para sostener que los nativos digitales son digitalmente competentes y que estas habilidades son transferidas al ámbito académico. A pesar de estar familiarizados con el uso de las tecnologías digitales y que sus habilidades digitales son altas, la competencia digital - ser capaz de explorar y enfrentar las nuevas situaciones tecnológicas de una manera flexible, para analizar, seleccionar y evaluar críticamente los datos e información; para aprovechar el potencial tecnológico con el fin de representar y resolver problemas, y construir conocimiento compartido y colaborativo, mientras se fomenta la conciencia de sus propias responsabilidades personales y el recíproco respeto de los derechos y obligaciones (Calvani, Cartelli, Fini \& Ranieri, 2008: 186) - puede ser menor que las de sus profesores. Si bien ahora se puede decir con certeza que la generación no es relevante (Bullen \& Morgan, 2011: 63), es necesario considerar otras variables a parte de la edad que pueden predecir la naturaleza del uso de las tecnologías digitales en los estudiantes.

Suponer que los estudiantes - quienes nacieron aproximadamente entre 1980 y 1994 -tienen habilidades digitales prácticamente naturales y no requieren una enseñanza formal, no debe ser absoluto. Según la revisión bibliográfica, la diversidad de estudiantes que hacen uso de la tecnología, tanto en su vida cotidiana así como en sus estudios académicos, nos indica que las generalizaciones basadas en conceptos generacionales de los nativos digitales no son útiles para discusiones sobre los cambios en estrategias de enseñanza y aprendizaje en la educación. Estas afirmaciones también deben incluir a los jóvenes menos hábiles en el uso de tecnologías, las condiciones en el acceso y uso de la información, y el descuido del impacto de factores de carácter contextual, económico, político, social, histórico y cultural que ahondan la brecha que se está abriendo entre quienes tienen acceso a la información y aquellos que no. Además en este campo, se ha evidenciado la carencia de estudios longitudinales así como de estudios en profundidad que documenten la 
naturaleza de sus habilidades y competencias, más allá de aspectos técnicos y que estén relacionados con sus procesos de aprendizaje.

Desde nuestro punto de vista para futuras investigaciones, es necesario reconocer el perfil del nuevo estudiante en los ingresantes a la universidad, analizando sus características específicas y tratar de desarrollar una comprensión global de los problemas que tengan en cuenta la diversidad de contextos culturales e institucionales (Bullen \& Morgan, 2011: 63). Además, consideramos importante realizar estudios más cualitativos para mejorar el aprendizaje de estos nativos digitales desde una perspectiva empírica. No cabe duda que son necesarias las investigaciones que nos aporten datos sobre cuál es el estado de la cuestión; es decir, cuál y cómo es el nivel de alfabetización de los estudiantes. Por lo tanto, es necesario tener en cuenta variables demográficas como el sexo, la universidad y el contexto cultural (Kennedy et al., 2010); disciplinas temáticas, diseño pedagógico de los cursos, situación socioeconómica de los estudiantes, sus circunstancias de vida, como por ejemplo, proximidad geográfica a sus amigos y familia, sociabilidad en general (extroversión, introversión) y así sucesivamente (Margaryan et al., 2011).

\section{Bibliografía}

Arafeh, S., Levin, D., Rainie, L., \& Lenhart, A. (2002). The digital disconnect: the widening gap between Internet-savvy students and their schools. Washington DC: Pew Internet \& American Life Project. Recuperado el 6 de febrero de 2012, de http://www.pewinternet.org/ /media//Files/Reports/2002/PIP Schools Internet Repo rt.pdf.pdf

Bajt, S. K. (2011). Web 2.0 Technologies: Applications for Community Colleges. Wiley Periodicals, Inc., (154), 53-62. doi:10.1002/cc.446

Ben-David Kolikant, Y. (2010). Digital natives, better learners? Students' beliefs about how the Internet influenced their ability to learn. Computers in Human Behavior, 26, 1384-1391. doi:10.1016/j.chb.2010.04.012

Bennett, S., Maton, K. \& Kervin, L. (2008). The 'digital natives' debate: a critical review of the evidence. British Journal of Educational Technology, 39, 5, 775-786. doi:10.1111/j.1467-8535.2007.00793.x

Brown, C., \& Czerniewicz, L. (2010). Debunking the "digital native": beyond digital apartheid, towards digital democracy. Journal of Computer Assisted Learning, 26(5), 357-369. doi:10.1111/j.1365-2729.2010.00369.x

Bullen, M. \& Morgan, T. (2011). Digital Learners not Digital Natives. La Cuestión Universitaria, 7, 60-68. 
Bullen, M.; Morgan, T., \& Qayyum, A. (2011). Digital Learners in Higher Education: Generation is Not the Issue. Canadian Journal of Learning and Technology, 37(1). Recuperado el 6 de marzo de 2012, de http://hdl.handle.net/10515/sy5wm1465

Cabero, J. Y Aguaded, J.I. (2003). Tecnologías en la era de la globalización. Revista Científica de Comunicación y Educación Comunicar, 21. Huelva: Grupo Comunicar.

Cabero, J. (2006, diciembre). La calidad educativa en el e-Learning: sus bases pedagógicas [en línea]. Educación Médica. 9(2), 7-12. Fundación Privada Educación Médica y Viguera Editores S.L.

Calvani, A., Cartelli, A., Fini, A., \& Ranieri, M. (2008). Models and Instruments for assessing Digital Competence at School. Journal of E-Learning and Knowledge Society, 4, 3. Recuperado el 12 de febrero de 2012, de http://jeIks.maieutiche.economia.unitn.it/index.php/Je-LKS EN/article/view/288

Camacho, M. M.; \& González, V. (2008). Desafíos de la educación preescolar en la era digital. InterSedes: Revista de las Sedes Regionales, 9(16), 69-88.

Coomes, M. D., \& Debard, R. (2004). A generational approach to understanding students. In M. D. Coomes \& R. DeBard (Eds.), Serving the millennial generation: New directions for student services, 106, 5-16. San Francisco: Jossey-Bass. doi: $10.1002 /$ ss.121

Corrin, L., Lockyer, L. Y Bennett, S. (2010). Technological diversity: An investigation of students' technology use in everyday life and academic study. Learning, Media and Technology, 35(4), 387-401.

D'amico, T. (2010-2011, Winter). Embracing 21st Century Technology. Leaders \& Learners. The official magazine of the Canadian Association of School Administrators, 29-31. Recuperado el 14 de marzo de 2012, de http://www.casaacas.ca/magazines/LL\%20MAg\%202010-2011\%20Winter.pdf.

Djamasbi, S., Siegel, M. \& Tullis, T. (2010). Generation Y, web design, and eye tracking. International Journal of Human-Computer Studies, 60, 307-323. doi:10.1016/j.ijhcs.2009.12.006

Frand, J. (2000). The information-age mindset: changes in students and implications for higher education. EDUCAUSE Review, 35, 14-24.

Gaston, J. (2006). Reaching and teaching the digital natives. Library Hi Tech News, 23, 3, 12-13. doi:10.1108/07419050610668124

Gisbert, M. \& Esteve, F. (2011). Digital learners: la competencia digital de los estudiantes universitarios. La Cuestión Universitaria, 7, 48-59.

Harel, I. (2003). Clickerati kids: Who are they? Recuperado el 11 de enero de 2012, de http://www.mamamedia.com/areas/grownups/new/21 learning/main.html 
Henry J. Kaiser Family Foundation (2005). Generation M: Media in the Lives of 8-18 Year Olds. Washington: Autor. Recuperado el 19 de marzo de 2012, de http://www.kff.org/entmedia/8010.cfm

International Learning Advisory Board (2008). Learning in the 21st Century: Teaching Today's Students on Their Terms. IEAB. Recuperado el 19 de marzo de 2012, de http://www.certiport.com/Portal/Common/DocumentLibrary/IEAB Whitepaper040808 .pdf

Irigoyen, O. (2000a). La era digital (I): ¿Sobran los maestros? Revisa La Onda Digital, 14. Recuperado el 5 de marzo de 2012, de http://www.laondadigital.com/LaOnda/LaOnda/001100/14/la\%20era\%20digital\%20i.htm

Irigoyen, O. (2000b). La era digital (II): Aprender a aprender. Revisa La Onda Digital, 14. Recuperado el 5 de marzo de 2012, de http://www.laondadigital.com/laonda/laonda/001100/15/Aprender\%20a\%20aprender.htm

Jones, C. \& Czerniewicz, L. (2010). Describing or debunking? The net generation and digital natives. Journal of Computer Assisted Learning, 26(5), 317-320. doi:10.1111/j.1365-2729.2010.00379.x

Jones, C., Ramanau, R., Cross, S. \& Healing, G. (2010). Net generation or Digital Natives: Is there a distinct new generation entering university? Computers and Education, 54(3), 722-732. doi:10.1016/j.compedu.2009.09.022

Jukes, I. (2009). Attributes of Digital Learners. Kelowna, BC, Canada: 21st Century Fluency Project.

Jukes, I.; Mccain, T.; \& Crockett, L. (2010). Understanding the Digital Generation: Teaching and Learning in the New Digital Landscape. Kelowna, BC, Canada: 21st Century Fluency Project.

Kennedy, G., Judd, T., Dalgarnot, B. \& Waycott, J. (2010). Beyond natives and immigrants: Exploring types of net generation students. Journal of Computer-Assisted Learning, 26(5), 333-343. doi: 10.1111/j.1365-2729.2010.00371.x

Kennedy, G., Judd, T. S., Churchward, A., Gray, K. \& Krause, K. (2008). First year students' experiences with technology: are they really digital natives? Australasian Journal of Educational Technology, 24(1), 108-122. Recuperado el 18 de noviembre de 2011, de http://www.ascilite.org.au/ajet/ajet24/kennedy.pdf

Kolikant, Y. (2010). Digital natives, better learners? Students' beliefs about how the Internet influenced their ability to learn. Computers in Human Behavior 26(6). doi:10.1016/j.chb.2010.04.012

Li, Y., \& Ranieri, M. (2010). Are 'digital natives' really digitally competent?-A study on Chinese teenagers. British Journal of Educational Technology, 41(6). doi:10.1111/j.1467-8535.2009.01053.x 
Margaryan, A., Littlejohn, A., \& Vojt, G. (2011). Are digital natives a myth or reality? University students' use of digital technologies. Computers \& Education, 56(2), 429440. Elsevier Ltd. doi:10.1016/j.compedu.2010.09.004

New York Library Association (2008). 21st Century Information Literacy Standards for the Digital Learners of New York. New York: NYLA. Recuperado el 15 de marzo de 2012, de http://thenewmiddleschool.files.wordpress.com/2010/02/digital-learnerstandards.pdf

Norum, P. S. (2008) "The role of time preference and credit card usage in compulsive buying behavior." International Journal of Consumer Studies, 32(3), 269-275. doi: 10.1111/j.1470-6431.2008.00678.x

Oblinger, D. G., \& Hawkins, B. L. (2005). The myths about students. Educause Review, 40(5), 12-13. Recuperado el 31 de enero de 2012, de http://net.educause.edu/ir/library/pdf/ERM0558.pdf

Oblinger, D. G., And Oblinger, J. L. (2005). Educating the Net Generation, Boulder, Colo.: Educause.

Palfrey, J., Gasser, U., Simun, M., \& Barnes, R.F. (2009). Youth, creativity and copyright in the digital age. International Journal of Learning \& Media, 1(2), 79-97. doi:10.1162/ijlm.2009.0022

Prensky, M. (2001a). Digital natives, digital immigrants, Part 1. On the Horizon, 9(5), 16. doi:10.1108/10748120110424816

Prensky, M. (2001b). Digital natives, digital immigrants, Part II: Do they really think differently? On the Horizon, 9(6), 1-9.

Prensky, M. (2005, December/ 2006, January). Listen to the Natives. Educational Leadership, 63(4), 8-13.

Prensky, M. (2007). How to teach with technology: Keeping both teachers and students comfortable in an era of exponential change. In Becta (Ed.), Emerging Technologies for Learning, 2, 40-46. British Educational Communications and Technology Agency.

Prensky, M. (2010). Teaching Digital Natives: Partnering for Real Learning. London: Corwin.

Rushkoff, D. (2006). ScreenAgers. Lessons in chaos from Digital Kids. New York: Hampton Press.

Tapscott, D. (1998). Growing up digital: the rise of the Net generation. New York: McGraw-Hill.

Taylor, P. And Keeter, S. (EDS.) (2010). Millennials: A portrait of generation next, Washington, DC.: Pew Research Center. 
United Nations Educational, Scientific and Cultural Organization (2011). Educación de calidad en la era digital: Una oportunidad de cooperación para UNESCO en América Latina y el Caribe. Documento interno de trabajo para la Reunión Regional Ministerial para América Latina y el Caribe, 12 y 13 de mayo de 2011. Santiago de Chile: Oficina Regional de Educación para América Latina y el Caribe/UNESCO. Recuperado el 31 de enero de 2012,

http://www.un.org/en/ecosoc/newfunct/pdf/4.desafios.para.la.educacion

Virkus, S. (2008). Use of Web 2.0 technologies in LIS education: experiences at Tallinn University, Estonia. Program: electronic library and information systems, 42(3), 262274. doi:10.1108/00330330810892677

Weiler A. (2005) Information-seeking behavior in generation-Y students: motivation, critical thinking and learning theory. The Journal of Academic Librarianship 31, 46-53. doi: 10.1016/j.acalib.2004.09.009

\section{Nota biográfica}

Eliana E. Gallado Echenique. Licenciada en Ciencias de la Comunicación. Master en Tecnologías Educativas: e-learning y gestión del conocimiento. Actualmente es estudiante de doctorado en Tecnologías Educativas y es becaria FPI de la Generalitat de Catalunya al Departamento de Pedagogía de la Universidad Rovira i Virgili. Miembro del grupo de investigación ARGET: Applied Research Group in Education and Technology (Ref. 2009SGR596). Además, ha participado en el diseño y la puesta en práctica de un sin número de proyectos de investigación y programas relacionados con las tecnologías de la información y la comunicación aplicadas a la educación, elaboración de materiales educativos y desarrollo de cursos de formación del profesorado.

E-mail: elianaesther.gallardo@urv.cat 